\title{
Ein juckendes Vergnügen
}

\section{An Itching Pleasure}

\author{
Gioia Braun-Meyre ${ }^{1}$, Corinne Marbet ${ }^{2}$, Marcel Blickenstorfer ${ }^{2}$ und Hamid Khani \\ ${ }^{1}$ Medizinische Universitätsklinik, Kantonsspital Baselland, Laufen \\ ${ }^{2}$ Klinik für Dermatologie, Universitätsspital Basel
}

Zusammenfassung: Eine 50-jährige Patientin stellt sich mit einem akneiformen, stammbetonten und stark juckenden Exanthem seit zwei Tagen sowie einem allgemeinen Krankheitsgefühl notfallmässig vor. Laboranalytisch zeigte sich keine Auffälligkeit. In der weiteren Anamnese gab die Patientin eine regelmässige Nutzung des neuen Whirlpools an. Aufgrund der Anamnese und Klinik wurde die Verdachtsdiagnose einer Pseudomonas-Follikulitis gestellt. Die mikrobiologische Untersuchung des Pustelinhalts ergab ein Wachstum von Pseudomonas aeruginosa, was den Verdacht bestätigte. Bei Abwesenheit von Risikofaktoren für einen schweren Verlauf wurde auf eine systemische Antibiotika-Therapie verzichtet. Unter externen Desinfektiva heilte die Dermatitis in wenigen Tagen komplikationslos aus.

Schlüsselwörter: Whirlpool-Dermatitis, Pseudomonas, Follikulitis

Abstract: A 50-year-old female patient presents in the emergency department with an itchy, acneiform exanthema on the trunk and malaise for two days. Laboratory testing showed no abnormalities. After further questioning, the patient reported frequent use of her new whirlpool. Based on the medical history and the clinic we suspected a pseudomonas folliculitis. A bacterial culture of the pustules showed growth of Pseudomonas aeruginosa, which confirmed the diagnosis. Systemic antibiotics were not necessary in the absence of risk factors for severe progress or immunosuppression. The exanthema healed within a few days without any complications by using external disinfectants.

Keywords: Hot-tub dermatitis, pseudomonas, folliculitis

\section{Anamnese und Befunde}

Eine 50-jährige Frau stellte sich im Frühjahr mit einem juckend-brennenden, stammbetonten Exanthem seit zwei Tagen auf unserer interdisziplinären Notfallstation vor. Trotz eines generellen Unwohlseins mit Fiebergefühl waren die Vitalparameter bei Eintritt einschliesslich der Temperatur im Normbereich. Die Patientin verneinte die Anwendung neuer Topika. Es bestanden keine relevanten Vorerkrankungen und keine regelmässige Medikamenteneinnahme. Die weitere Anamneseerhebung ergab eine tägliche Nutzung eines neu gekauften Whirlpools, der gewissenhaft mit chlorhaltigen Desinfektionsmitteln gereinigt werde. In der körperlichen Untersuchung zeigte sich ein akneiformes Exanthem mit follikulär gebundenen erythematösen Papeln und Pusteln (Abb. 1), das sich über dem ventralen und dorsalen Stamm unter Aussparung der Extremitäten und des Kopfes sowie der Schleimhäute beobachten liess. Der übrige Status gestaltete sich unauffällig, insbesondere fehlten eine Lymphadenopathie oder weitere Organpathologien. Laborchemisch fand sich ein leicht erhöhtes C-reaktives Protein von $20 \mathrm{mg} / \mathrm{l}$, das restliche Routinelabor mit Leukozyten $(8,1 \mathrm{G} / \mathrm{l})$, Leber- und Nierenretentionsparametern war unauffällig.

\section{Weitere Abklärungsschritte und Verlauf}

Weiterhin berichtete die Patientin von einer erstmaligen subkutanen Vitamin-B12-Gabe drei Tage zuvor. Zur weiteren Abklärung einer möglichen mikrobiologischen Ätio-

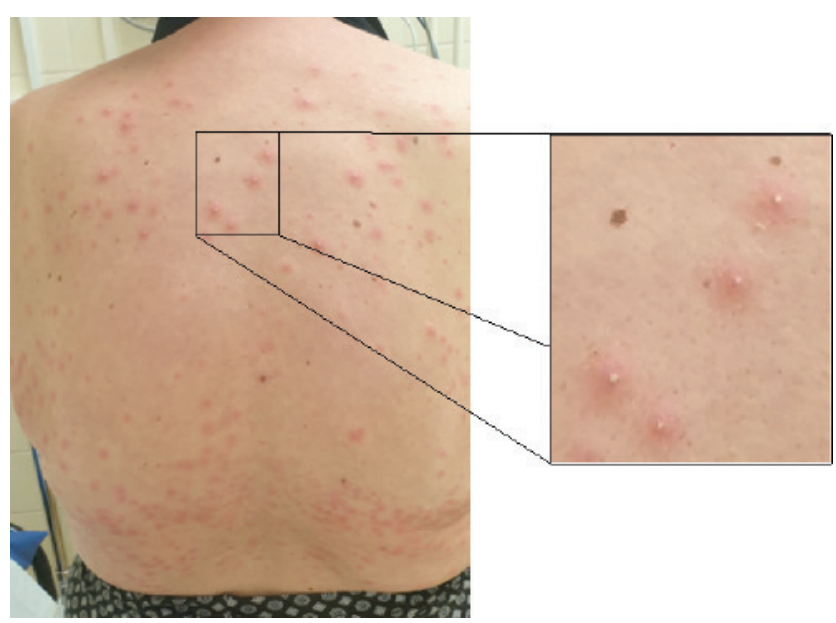

Abbildung 1. Akneiformes Exanthem, mit follikulär gebundenen erythematösen Papeln und Pusteln, stammbetont. 
logie erfolgte die bakteriologische Untersuchung des Pustelinhalts. In der Gram-Färbung zeigten sich Gramnegative Stäbchen.

\section{Differenzialdiagnostische Überlegungen}

Aufgrund der vorliegenden Befunde, insbesondere der makroskopischen Hautveränderungen, stand die Verdachtsdiagnose einer infektiösen Follikulitis im Vordergrund.

Differenzialdiagnostisch käme auch eine Akne vulgaris in Frage, allerdings sind die Verteilung der Hautläsionen und die klinische Präsentation sowie das Alter der Erstmanifestation untypisch. Des Weiteren wären Miliaria, Skabies, Pyodermie, Prurigo simplex subacuta, Varizellen oder andere Virusexantheme möglich. Ein Prurigus simplex subacuta ist bei atypischem Alter der Patientin bei Erstmanifestation sowie der ausbleibenden Linderung des Juckreizes nach Kratzen eher auszuschliessen. Aufgrund des stammbetonten Verteilungsmusters unter Aussparung der Extremitäten ist eine Skabies-Infektion eher unwahrscheinlich. Eine Varizellen-Infektion geht typischerweise mit Befall der Schleimhäute einher. Die Miliaria ist nicht follikulär gebunden und kann aufgrund des Krankheitsauftretens in der kühlen Jahreszeit eher ausgeschlossen werden. Als weitere Alternative ist ein akneiformes Exanthem nach Vitamin-B12-Gabe bekannt, was zur klinischen Präsentation passen würde.

\section{Diagnose}

In der angelegten Kultur des Abstriches des Pustelinhalts liess sich ein Wachstum eines pansensiblen Pseudomonas aeruginosa nachweisen. In Zusammenschau von Anamnese, Klinik und mikrobiologischen Befunden konnte die Diagnose einer Hot-Tub-Dermatitis/Pseudomonas-Follikulitis gestellt werden. Ein antiseptisches Externum mit Chlorhexidindiglukonat wurde rezeptiert. Bei gutem Allgemeinzustand sowie fehlenden Risikofaktoren für einen schweren Verlauf (Immunsuppression, schwere Grunderkrankung, etc.) wurde auf eine systemische Therapie verzichtet. In der Verlaufskontrolle nach zehn Tagen zeigte sich erfreulicherweise ein deutlich regredienter Verlauf. Die Follikulitis heilte im Verlauf vollständig aus.

\section{Kommentar}

Die Ursache der Pseudomonas-Follikulitis liegt in einer bakteriellen Infektion der Haarfollikel nach Exposition mit kontaminiertem Wasser, häufig von Whirlpools [1] Die exakte Inzidenz der Pseudomonas-Follikulitis ist nicht bekannt. Patientinnen und Patienten mit Diabetes mellitus, Adipositas, prolongierter Antibiotika-Einnahme oder Immunsuppression haben ein höheres Erkrankungsrisiko sowie ein höheres Risiko eines schweren Verlaufes [2]. Ein erster grosser Ausbruch in einem öffentlichen Schwimmbad in Amerika wurde 1983 beschrieben [3]. Aufgrund der typischen Hautveränderungen mit assoziierter Warmwasser-Nutzung ist vor allem in den Wintermonaten an diese Diagnose zu denken.

Komplikationen sind nicht selten. Die bakterielle Infektion kann sich auf andere Organsysteme ausweiten, was sich als Otitis externa, Konjunktivitis, Mastitis sowie eine regionäre Lymphadenitis manifestieren kann.

Die Pseudomonas-Follikulitis ist vergesellschaftet mit einer hohen Infektiosität. Auch der Sohn unserer Patientin nutzte den Whirlpool regelmässig und zeigte wenige Tage später die gleichen Hautveränderungen sowie eine Otitis externa als Komplikation.

Wie in unserem Fall, reicht in leichten Fällen meist eine regelmässige äusserliche Desinfektion zur Heilung. Falls nach 10-14-tägiger Frist keine Besserung der Hautveränderungen zu erreichen ist oder Komplikationen auftreten, sollte eine lokale oder systemische Therapie mit Ciprofloxacin in Erwägung gezogen werden [4].

Studien haben gezeigt, dass in $21 \%$ aller Wasser- und Abstrich-Proben aus Whirlpools Pseudomonas aeruginosa nachgewiesen werden konnte. 96\% der abgenommenen Kulturen waren multiresistent, auch gegen Chlor [5]. Diese Beobachtung erschwert die Wahl eines wirkungsvollen Reinigungsmittels. Interessanterweise hat das Abwaschen des kontaminierten Wassers nach der Whirlpool-Benutzung keinen signifikanten protektiven Effekt [6]. Die Dauer der Exposition mit kontaminiertem Wasser sowie eine spätabendliche Nutzung des Whirlpools sind mit einem höheren Erkrankungsrisiko assoziiert [7].

Die Sauberhaltung des Whirlpools ist essenziell zur Vermeidung dieser meist ungefährlichen, aber äusserst unangenehmen Erkrankung.

\section{Bibliografie}

1. Gregory DW, Schaffner W. Pseudomonas infections associated with hot tubs and other environments. Infect Dis Clin North Am. 1987;1(3):635-648.

2. Winters RD, Mitchell M. Folliculitis. StatPearls [Internet]. Treasure Island (FL): StatPearls Publishing; 2019 Sep 18 [cited

Key messages

- Die Pseudomonas-Follikulitis ist eine gutartige, selbstlimitierende, infektiöse Hauterkrankung, verursacht durch Pseudomonas aeruginosa.

- Eine systemische Therapie ist bei Abwesenheit von Risikofaktoren wie Immunsuppression oder schwerer Grunderkrankung oft nicht notwendig. Bei Bedarf kann eine antibiotische Therapie mit Ciprofloxacin initiiert werden 
2020 Jan]. https://www.ncbi.nlm.nih.gov/books/NBK547754/; letzter Zugriff: 01.10.2020.

3. Gustafson TL, Band JD, Hutcheson RH Jr, Schaffner W. Pseudomonas folliculitis: an outbreak and review. 1983;5:1-8.

4. Dietrich KA, Ruzicka T, Herzinger T. [Whirlpool-dermatitis with «hot hands»]. Dtsch Med Wochenschr. 2014;139(28-29): $1459 \mathrm{c} 61$.

5. Lutz JK, Lee J. Prevalence and antimicrobial-resistance of Pseudomonas aeruginosa in swimming pools and hot tubs. Int J Environ Res Public Health. 2011;8(2):554-564.

6. Yu Y, Cheng AS, Wang L, Dunne WM, Bayliss SJ. Hot tub folliculitis or hot hand-foot syndrome caused by Pseudomonas aeruginosa.J Am Acad Dermatol. 2007;57(4):596-600.

7. Kosatsky T, Kleeman J. Superficial and systemic illness related to a hot tub. Am J Med. 1985;79(1):10.

\section{Historie}

Manuskript eingereicht: 22.08.2020

Nach Revision angenommen: 22.09.2020

\section{Interessenskonflikt}

Die Autorinnen und Autoren erklären, dass keine Interessenskonflikte bestehen.

\section{Dr. med. Gioia Braun-Meyre}

Rheinstrasse 26

4410 Liestal

gioia-danae.braun@ksbl.ch 